\title{
LA SALUD DE MUJERES MIGRANTES LATINOAMERICANAS EN LA ENCRUCIJADA ENTRE LOS CUIDADOS REMUNERADOS Y NO REMUNERADOS: UN ANÁLISIS DESDE SUS NARRATIVAS DE AFLICCIÓN
}

\author{
JULIETA MAURE \\ Máster Interuniversitario en Antropología Médica y Salud Clobal \\ Universitat Rovira i Virgili \\ Julymaure22@gmail.com
}

RESUMEN: La salud de mujeres latinoamericanas que trabajan como cuidadoras en Barcelona atraviesa una multiplicidad de violencias y desigualdades, favorecidas por políticas de Estado estructuralmente patriarcales y coloniales. La explotación laboral que padecen, a consecuencia de la ilegalidad en que coloca la legislación española a muchas de ellas, coarta su autonomía y las obliga a optar por el trabajo de cuidados en régimen de internamiento, lo que implica una convivencia jerarquizada y en situación de encierro en el hogar de la persona a la que cuidan. A su vez, las mujeres afrontan las constricciones y dificultades que implican las tareas de cuidado que realizan a nivel transnacional, asegurando la supervivencia de sus hijas, hijos y otros familiares en el país de origen. A través de entrevistas en profundidad realizadas a seis mujeres latinoamericanas que trabajan como cuidadoras en Barcelona, el presente trabajo se acerca a esta realidad social, política y económica que implica la Fuga de Cuidados (Bettio, Simonazzi, \& Villa, 2011) del sur al norte global, a partir de las vivencias y narrativas de aflicción de mujeres latinoamericanas que migran para cuidar, en un contexto en donde la accesibilidad real al sistema sanitario se encuentra restringida.

PALABRAS CLAVE: migración femenina; cuidadoras; maternidad transnacional; salud; antropología médica 
ABSTRACT: The health of Latin American women who work as caregivers in Barcelona is conditioned by multiple forms of violence and inequalities, favoured by structurally patriarchal and colonial state policies. The labour exploitation that they suffer, as a consequence of the illegality in which the Spanish legislation places many of them, limits their autonomy and forces them to opt for a job as caregivers in a 'live in' regime, which implies a hierarchical coexistence in a situation of confinement with the person they work for. In addition, women face the constraints and difficulties involved in the caregiving tasks they perform at the transnational level, ensuring the survival of their daughters, sons and other relatives in the country of origin. Through in-depth interviews with six Latin American women who work as caregivers in Barcelona, the present study approaches this social, political and economic reality of the Care Drain (Bettio, Simonazzi, \& Villa, 2011) from the global south to the north, based on the experience and illness narratives of Latin American women who migrate to perform caregiving tasks, in a context where real accessibility to the health system is restricted.

KEYWORDS: female migration; caregivers; transnational mothering; health; medical anthropology 


\section{Introducción}

A la larga lista de materias primas que desde hace siglos los países del norte vienen extrayendo de los países del sur - y que otrora encabezaron el oro y la plata-, en nuestros días se le suma una forma no tan novedosa de extractivismo: el del afecto y el cuidado. Esta 'materia prima', fundamental para la vida y el desarrollo de los seres humanos, es el «nuevo oro» (Hochschild, 2002) puesto desde América Latina al servicio de las necesidades de los "países desarrollados» desde hace décadas. Mujeres, en su mayoría empobrecidas, son conducidas a atravesar fronteras en busca de mejores oportunidades para asegurar su propia supervivencia y la de su familia o comunidad. El desplazamiento hacia la antigua metrópoli se muestra prometedor, tanto por las facilidades del idioma como por la gran demanda de trabajo que llega a sus oídos, e incluso antes de que arriben a destino las mujeres son conscientes de que tienen un sector laboral esperando por ellas: el trabajo de hogar y de cuidados.

Una vez que llegan a España, se encuentran con la enorme demanda de cuidado de personas mayores. Las familias las buscan específicamente para este trabajo porque las consideran educadas, tranquilas, pacientes, cariñosas y agradecidas ${ }^{1}$. Quieren que sus madres y padres, ya entrados en años, frágiles y con varios problemas de salud, sean acompañados y cuidados por ellas, presentándose como una opción mucho más amigable que enviarlos a una residencia. A pesar de los bajísimos salarios y del régimen de explotación que les ofrecen, las mujeres recién migradas no tienen muchas posibilidades de rechazarlos, ya que la ley de extranjería del Estado español impone fuertes restricciones a la obtención del permiso de residencia y de trabajo, lo que sitúa a muchas de ellas en situación ilegal (Ley Orgánica 4, 2000). Esto las condiciona a entrar y mantenerse en la economía sumergida, reforzando así la desigualdad en la relación de poder entre el empleador y la trabajadora, por lo que acaban trabajando en condiciones donde se les niega de facto el derecho a la autonomía. Esta situación de ilegalidad hace que las trabajadoras se enfrenten a mayores riesgos y cargas (Hondagneu-Sotelo, 2001) y representa, a su vez, el principal obstáculo para un adecuado acceso al sistema sanitario. 
A las dificultades derivadas de sus historias de empobrecimiento, de violencia machista o conflictos familiares, a muchas mujeres se les suman las constricciones por verse obligadas a dejar a sus hijos en el país de origen al momento de emigrar. Empero, esto no se traduce para ellas en la interrupción de sus tareas y responsabilidades como madres, ya que a pesar de la distancia, continúan siendo las principales responsables del desarrollo de los 'suyos' en origen y las encargadas de asegurar su supervivencia. En este sentido, las prácticas de maternidad transnacional (Herrera, 2010), en donde las mujeres se adaptan a las nuevas formas de cuidado de los hijos e hijas a distancia, se despliegan en simultaneidad con el cuidado de ancianos, que realizan como actividad remunerada.

El lugar que ocupan estas mujeres como cuidadoras bilocales o multilocales tiene múltiples efectos sobre su salud, ya que recae sobre sus cuerpos una intersección de violencias que sirven de reflejo de un sistema global que reproduce y multiplica las desigualdades. El presente trabajo intenta un acercamiento a esta realidad social, política y económica transnacional que representa la Fuga de Cuidados (Bettio, Simonazzi, \& Villa, 2011) desde el sur al norte global, enfocada desde lo que ocurre en el lado europeo de esta problemática —específicamente en la ciudad de Barcelona- a partir de las narrativas de aflicción de mujeres latinoamericanas que migran para dedicarse a cuidar. En este sentido, mi propia condición de mujer migrante, también latinoamericana, me permitió alcanzar una empatía necesaria con mis informantes, descontando las diferencias que nos separaban por mis privilegios al tener orígenes europeos (el llamado 'capital de la blancura') y mi desarrollo profesional. Por otra parte, mi formación como profesional de la salud me permitió un 'ir y venir' en la interpretación de sus relatos, entre la enfermedad y la aflicción, mediado por una escucha antropológica, poniendo en diálogo los saberes médicos y el saber que aporta la experiencia vivida. 


\section{Marco teórico}

\subsection{Los cuidados y la reproducción social}

A partir de la industrialización y la consolidación de los Estados modernos se produjo una reconfiguración de la división sexual del trabajo, estableciéndose sobre dicotomías en términos de esferas o ámbitos (público / privado), valor económico (remunerado / no remunerado) y valor intelectual (profesional / no profesional), entre otras. Las mujeres, restringidas al ámbito doméstico, fueron las que sostuvieron las tareas de atención y cuidado de las personas dependientes, ya sea en papel de madre, hermana, tía, abuela, vecina, etc. Estas tareas, llevadas a cabo en el contexto de las relaciones familiares establecidas por el parentesco, han sido socialmente concebidas como productos «naturales» del amor maternal, no como trabajo que debe ser remunerado. La vinculación entre los cuidados y «el amor» ha contribuido a la desvalorización de estas tareas (Comas d'Argemir, 1995), y a la apropiación y ocultamiento del trabajo de las mujeres por parte de la institución familiar (Federici, 2018). Dentro de la organización de la vida humana, este conjunto de tareas constituye una parte de la esfera de la reproducción social, entendida esta como «un complejo proceso de tareas, trabajo y energías, cuyo objetivo sería la reproducción de la población, y de las relaciones sociales, y en particular, la reproducción de la fuerza de trabajo» (Carrasco, Borderías, \& Torns, 2011: 31).

En las sociedades europeas, la entrada masiva de las mujeres de clase media al mercado laboral en las últimas décadas del siglo xx ocasionó un quiebre en la forma tradicional de organizar el cuidado dentro de las familias. Esta merma tan acusada en la disponibilidad de tiempo de quienes eran las principales agentes de cuidado, sumado a un progresivo envejecimiento de la población y, por ende, un crecimiento de la población dependiente,la falta de participación masculina en estas tareas y el debilitamiento de las redes comunitarias de cuidado, comenzaron a poner en riesgo la reproducción social y la sostenibilidad del sistema capitalista. El problema vertebrador del presente trabajo se sitúa en esta crisis de la reproducción social, y más específicamente en la Crisis de los Cuidados (Comas d'Argemir, 2014) en el contexto específico de Cataluña y el conjunto del Estado español, puesto que se constituye como aquello que le da senti- 
do a la experiencia migratoria, laboral y vital de las mujeres de mi estudio, quienes emigraron, en parte, a causa de esta gran demanda de cuidados.

Aunque el trabajo de cuidados no es fácil de definir y ha sido objeto de intensos debates dentro del feminismo, podemos pensarlo en términos generales como una forma de proveer bienestar, tanto físico, psíquico, como emocional entre seres humanos (Comas d'Argemir, 2014). Esta definición, si bien más abstracta, considera el concepto de bienestar como finalidad, y permite contemplar la inmensa inversión de tiempo y energía que socialmente se espera de las mujeres para mantener la comodidad, felicidad y cohesión entre los miembros de un grupo social, sea dentro o fuera de la unidad familiar.

Sin embargo, desde el feminismo académico no todas las autoras concuerdan con la utilización tan amplia del concepto 'cuidados'. En el texto Los cuidados, un concepto central en la teoría feminista: aportaciones, riesgos y diálogos con la antropología, Mari Luz Esteban (2017) advierte sobre el sobredimensionamiento del término y la necesidad de tener en cuenta sus connotaciones históricas, culturales y de género. La antropóloga prefiere hacer un uso más limitado de este concepto y se inclina por la utilización de otros, como el de 'atención a la dependencia', la cual define como aquellas atenciones que se brindan «a todas las personas que temporal o definitivamente no pueden valerse por sí mismas por razones de edad, enfermedad o condición» (2017: 41). En relación con este debate, concuerdo con Dobrée en afirmar que «la mayor o menor amplitud que se atribuye al concepto tiene importantes implicancias filosóficas y pragmáticas» (2016: 174). Desde mi perspectiva, la 'atención a la dependencia' que propone Esteban no acaba de reflejar todo el trabajo y las energías exigidas a las mujeres para asegurar el bienestar de las personas que no podrían considerarse dependientes. ¿Es dependiente un marido que no cocina?, ¿o un hijo de 20 años que no se lava su ropa? Dejar por fuera de la definición, y por ende del análisis, a estas energías invertidas impide comprender en profundidad los efectos del trabajo de cuidados en la salud. En este sentido, observamos que son escasos en la literatura los estudios que consideren los efectos de ambos tipos de trabajo de cuidados, los remunerados y los no remunerados, en la salud de las trabajadoras. 


\subsection{La salud y la enfermedad: contribuciones desde la antropología médica}

La antropología médica, en toda su heterogeneidad y principalmente a partir del método etnográfico, ha sabido contribuir al conocimiento sobre una dimensión tan compleja de la vida humana como lo es la salud, la enfermedad y los sistemas médicos. A partir de la escisión del paradigma ciencia frente a creencia que caracterizó al modelo clásico (Martínez Hernáez, 2015) se dejó de considerar a la medicina como único paradigma científicamente válido para abordar la salud y la enfermedad, entendiéndola como uno más dentro de los sistemas médicos existentes, si bien es dueña de la hegemonía del saber reconocido sobre la salud y la enfermedad en sociedades occidentales. De esta forma, la medicina occidental pasó a ser objeto de estudio de la antropología —al igual que otros sistemas médicos - siendo criticado por las limitaciones de la ahistoricidad, biologicismo y asociabilidad en su enfoque de la salud humana (Menéndez, 2005).

Otra separación importante que la antropología médica desarrolló fueron las distintas dimensiones que constituyen la experiencia de la enfermedad, representadas por las distintas palabras que la definen en inglés (Illnes, Sickness, Disease). Arthur Kleinman, psiquiatra y antropólogo estadounidense, contribuyó a esta diferenciación mediante la conceptualización de los términos Illness y Disease (1978). Al concepto de Illness lo define como el padecimiento o aflicción de la persona enferma. Es la manifestación de los síntomas que, embebidos en mundos de significado, constituyen la expresión del malestar. Estas manifestaciones son, a su vez, distintivas y culturalmente moldeadas (Kleinman, 1988). Por otra parte, el concepto de Disease es la explicación biológica que brinda el médico respecto al padecimiento de quien en la relación clínica es su paciente. Luego, encontramos el concepto de Sickness, que representa el proceso de socialización del Illness o el Disease, aludiendo a la dimensión social, política y económica que hace posible la construcción y el reconocimiento de una patología determinada.

La biomedicina se ha centrado de manera casi exclusiva en la dimensión del Disease, y la antropología se ha visto más interesada en las otras dos dimensiones. Tenemos, por un lado, una antropología médica in- 
terpretativa, de tipo hermenéutica y enfocada en el Illness, cuyo objetivo central es la comprensión de esos mundos de significado presentes en las narrativas de aflicción. Por otra parte, la antropología médica crítica, de orientación predominantemente materialista, coloca el eje en el Sickness, y entiende las aflicciones y enfermedades como resultado de unas determinadas relaciones de opresión política, influidas por factores macroeconómicos y sociales.

Este trabajo opta por una aproximación alternativa y complementaria que propone el antropólogo Ángel Martínez, quien plantea un abordaje interpretativo y necesariamente crítico de las narrativas de aflicción, entendiéndolas también como formas de expresión de desigualdades sociales, así como de relaciones de poder y dominación. Se trata de centrarse en el síntoma, no como disfunción orgánica, tal como lo establece la medicina, sino en el síntoma como mensaje, como texto, como discurso. También adentrarse en el Illness sin perder de vista el Sickness, enfocarse más en comprender que en explicar, y en esa comprensión no perder de vista las formas de opresión política, ni tampoco ignorar o silenciar la voz de la persona que sufre, ya que esto puede dar lugar a «una nueva y sutil forma de dominación» (Martínez-Hernáez, 2000: 175).

\section{Metodología}

La presente exposición fue elaborada a partir de la investigación que realicé para el Trabajo Final del Máster en Antropología Médica y Salud Global (URV-UB), el cual fue desarrollado con una metodología cualitativa. El trabajo de campo se llevó a cabo entre los meses de febrero y mayo de 2019, tiempo en el que se realizaron entrevistas individuales en profundidad, tipo historia de vida, a seis mujeres migrantes latinoamericanas que trabajan o trabajaron en algún momento cuidando a personas en situación de dependencia en Barcelona. Todas las informantes se encontraban residiendo en Barcelona, todas trabajaron o se encontraban trabajando de internas en el momento de las entrevistas.

El contacto con las informantes se estableció mediante enfermeras y médicas del Centro de Atención Primaria donde me encontraba ejerciendo como médica de manera temporal. Si bien las mujeres entrevistadas 
estaban informadas de mi profesión, mi intención desde un principio fue contactarlas fuera de la consulta e idealmente a través de otros profesionales del centro, para que no confundieran mi rol de investigadora con el de médica. Además, quise transmitirles que la investigación en curso no tenía ningún vínculo con la institución sanitaria en cuestión. Sin embargo, a Sandra y Catalina las conocí personalmente en la consulta médica, y decidí invitarlas a participar en el estudio porque consideré que sus experiencias vitales resultaban de suma importancia para la investigación, aunque sabía que corría el riesgo de que se diera una confusión de roles. Aun así, todas las entrevistas se llevaron a cabo fuera del entorno sanitario: algunas de ellas se hicieron en plazas públicas, otras en el domicilio particular o en el lugar de trabajo (para quienes trabajaban de internas, estos dos lugares coincidían) y otras en un café de la ciudad.

Fue necesario orientar las entrevistas de manera biográfica para comprender la vivencia de sus aflicciones, lo cual me permitió conocer en profundidad el contexto transnacional en el cual se encontraban insertas. En las entrevistas procuré abordar las distintas etapas del proceso migratorio. La vida en el país de origen - situación socioeconómica, vínculos familiares, relaciones de género, trabajo, salud-, el proceso y los motivos que las llevaron a tomar la decisión de emigrar, así como la experiencia vivida en el país de destino. Sobre la etapa en el país de destino, se abordaron los mismos aspectos mencionados respecto al país de origen, aunque poniendo especial énfasis en la modalidad de trabajo de cuidados, los cuidados transnacionales y la salud. 


\begin{tabular}{|l|c|l|}
\hline Nombre & $\begin{array}{c}\text { Añode } \\
\text { llegadaa } \\
\text { Barcelona }\end{array}$ & \multicolumn{1}{|c|}{ Resumen biográfico } \\
\hline $\begin{array}{l}\text { Elisabeth } \\
(32)\end{array}$ & 2017 & $\begin{array}{l}\text { Ciudad del Este, Paraguay. Secundario completo. Soltera. Una } \\
\text { hija (7a) en Paraguay a cargo de su hermana. Trabaja de inter- } \\
\text { na como cuidadora. Sin permiso de residencia. Envía remesas } \\
\text { para su hija. }\end{array}$ \\
\hline $\begin{array}{l}\text { Mónica } \\
(51)\end{array}$ & 1996 & $\begin{array}{l}\text { Cuenca, Ecuador. Universitario completo. Separada. Dos hi- } \\
\text { jos/as (22 y 19a) nacidos en Barcelona, actualmente viviendo } \\
\text { en Ecuador. Trabaja en limpieza de hogares por horas durante } \\
\text { la semana, y de interna como cuidadora los fines de semana. } \\
\text { En total 10o horas/semana. Con permiso de residencia y tra- } \\
\text { bajo. Envía remesas para sus hijos. }\end{array}$ \\
\hline $\begin{array}{l}\text { Sandra } \\
(57)\end{array}$ & 2002 & $\begin{array}{l}\text { Andahuaylas, Perú. Primario completo. Tres hijos/as (35, 32 y } \\
\text { 30a) en Brasil, Perú y Barcelona. Precaria situación socioeco- } \\
\text { nómica en origen. Marido alcohólico y maltratador en Perú. } \\
\text { Trabajó como cuidadora de interna muchos años, actualmen- } \\
\text { te trabaja de cuidadora por horas. Con permiso de residencia } \\
\text { y trabajo. Ha enviado remesas a sus hijos hasta su indepen- } \\
\text { dencia económica. }\end{array}$ \\
\hline $\begin{array}{l}\text { Catalina } \\
(65)\end{array}$ & 2003 & $\begin{array}{l}\text { Trinidad, Bolivia. Primario completo. En concubinato. Dos hi- } \\
\text { jos/as (32 y 22a) en Bolivia. Situación socioeconómica precaria } \\
\text { en origen. Ha trabajado en el sector de la limpieza, también de } \\
\text { interna como cuidadora, y abrió un negocio que fracasó. Con } \\
\text { permiso de residencia y trabajo. Envía remesas para su hija. }\end{array}$ \\
\hline $\begin{array}{l}\text { Lucía } \\
(48)\end{array}$ & 2010 & $\begin{array}{l}\text { Tegucigalpa, Honduras. Primario completo. Casada (marido } \\
\text { en Honduras). Cinco hijos/s (tres en Barcelona, uno en Hon- } \\
\text { duras, uno fallecido). Una hija con discapacidad. Ha trabajado } \\
\text { de interna como cuidadora hasta la actualidad. Con permiso } \\
\text { de residencia y trabajo. Envía remesas a su familia. }\end{array}$ \\
$\begin{array}{l}\text { Misiones, Argentina. Primario completo. Separada. Seis hijos/ } \\
\text { as mayores de edad en Argentina. Un hijo con problemática de } \\
\text { adicciones, varias internaciones. Emigró por crisis económica } \\
\text { de 20o1. Trabaja como de interna como cuidadora actualmen- } \\
\text { te. Ha trabajo en el sector de la limpieza y en casa de colonias. } \\
\text { Con permiso de residencia y trabajo. }\end{array}$ \\
\hline
\end{tabular}




\section{La salud y los cuidados}

Los estudios realizados en relación con la salud de mujeres trabajadoras del hogar y los cuidados aportan datos que no resultan sorprendentes. Además de los problemas musculoesqueléticos, la afectación de la salud mental suele ser uno de los hallazgos más comunes en la literatura, tal cual lo reflejó una revisión sistemática (Malhotra y otros, 2013), entre otros estudios. Los factores que se han identificado como los relacionados con una peor calidad de vida percibida tienen que ver con el hecho de estar 'de interna', la ausencia de un contrato laboral, el desarrollo de múltiples tareas, el estatus migratorio irregular y la edad más joven (Bover, y otros, 2015) (Ahonen, y otros, 2010) (Offenhenden, 2017). Además, la existencia de leyes que atentan contra la universalidad de la atención médica coloca a las mujeres migrantes como uno de los colectivos con menor acceso al sistema sanitario y, por lo tanto, con mayor probabilidad de enfermar, de no poder ejercer con plenitud sus derechos sexuales y reproductivos, y de no ser detectadas cuando sufren violencia machista (Morero Beltrán \& Ballesteros Pena, 2014).

\subsection{Del cuidado de personas mayores a una relación de dominación}

Los servicios de proximidad, y específicamente el cuidado de ancianos, son nichos laborales que ya estaban reservados para las mujeres de mi estudio (Parella, 2004) incluso antes de que ellas tomaran la decisión de emigrar. Cuando la gran demanda de agentes de cuidados se encuentra con la existencia de mujeres migrantes, racializadas, sin papeles, con pocos recursos económicos y escasas redes sociales, en un contexto legal que las deja desprotegidas, el resultado es una realidad que pude observar con enorme frecuencia, tanto en mi rol de médica como de investigadora: el régimen de internamiento. Este sistema funciona como una forma de convivencia jerarquizada en la cual las trabajadoras se encuentran viviendo bajo el mismo techo con la persona a la que cuidan, veinticuatro horas al día. Las tareas que se les demandan suelen ser de todo tipo y los límites a esas demandas suelen ser escasos y difusos, lo que provoca que finalmente sean las responsables de casi todo lo que ocurre en el domicilio. En palabras de Sandra: «interna quiere decir que me hago cargo de todo». 
La opción de estar 'de internas' suele ser la más tentadora, especialmente recién llegadas, teniendo en cuenta sus restringidas posibilidades en destino, además de ser la única alternativa que les evita tener que afrontar un alquiler imposible de pagar en una ciudad costosa como Barcelona. De esta manera también se evitan pagar gastos de comida o transporte, y así pueden maximizar los ahorros, ya sea para enviar remesas a sus familiares, pagar las deudas que contrajeron para poder viajar o cumplir con otros objetivos, como comprarse su propia casa en el país de origen. En este régimen de internamiento algunas tienen contrato, otras no; algunas tienen los fines de semana libres, otras trabajan de lunes a lunes; algunas tienen estipulados horarios de descanso, otras simplemente no. El encierro físico y el aislamiento social se convierte en la realidad de todos los días, con lo cual el imperativo médico de «salir a hacer ejercicio para bajar el colesterol» que reciben mis informantes, entre otros tantos consejos, se vuelve un imposible.

Las tareas de cuidado que suelen realizar fueron recogidas en el trabajo de campo según sus propios relatos y se encuentran clasificadas en la Tabla 1. Dividir y clasificar las tareas de cuidado es sumamente compleja: refleja la dificultad misma de definir qué son los cuidados y qué abarcan. La clasificación de la Tabla 1 busca exponer la diversidad de actividades que se esperan de ellas en el contexto del cuidado de ancianos, a la vez que ayuda a pensar en los posibles efectos sobre su salud y arroja luz sobre el rol que cumplen como efectoras del sistema sanitario ${ }^{2}$. Además, se propone visibilizar algunas actividades que socialmente no son tenidas en cuenta como parte del trabajo que realizan, y que por ende requieren de nuestra mayor atención e interés como investigadoras. Me refiero a las que he denominado como «tareas de proximidad» y que contemplan todas aquellas acciones que tienen como objetivo mismo la interacción y cercanía con la persona a la que se cuida, contribuyendo en gran medida al bienestar psíquico de esta última. Estas prácticas pueden ser tan sutiles como prestar atención a una historia o reír ante una broma que se cuenta. Sin embargo, aunque sutiles, requieren de una inversión de

2 «Yo les recalco que mi paciente es la persona que está sentada y que ella [la cuidadora] es como mi compinche para que la cosa vaya bien». Cristian. Enfermero. 
tiempo y energías que no deben subestimarse. Al igual que como ocurre con los cuidados de madres a hijos, en los que estas tareas son consideradas como parte del «amor maternal» y no como trabajo, en este caso es posible que las tareas de proximidad sean consideradas como producto de otro tipo de «amor» o «vocación» mistificada.

\section{Tabla 1. Clasificación de actividades basada en la función (tareas descriptas por mis informantes)}

\begin{tabular}{|l|l|l|}
\hline Categoría-Función & Tareas & $\begin{array}{l}\text { Ayudar a vestirse } \\
\text { Lavar la ropa } \\
\text { Planchar la ropa } \\
\text { De sostén cotidiano }\end{array}$ \\
& $\begin{array}{l}\text { Hacer las compras } \\
\text { Cocinar } \\
\text { Servir la cena y recoger } \\
\text { Lavar los platos } \\
\text { Ayudar a duchar } \\
\text { Acompañar al baño } \\
\text { Dejar lista la ropa }\end{array}$ & $\begin{array}{l}\text { la cama } \\
\text { Cambiar pañales } \\
\text { Limpiarlos }\end{array}$ \\
\hline $\begin{array}{l}\text { De proximidad } \\
\text { - Interactivas }\end{array}$ & $\begin{array}{l}\text { Hacer compañía } \\
\text { Escuchar historias } \\
\text { Reír de bromas } \\
\text { Conversar }\end{array}$ & $\begin{array}{l}\text { Llevar a pasear } \\
\text { Dar apoyo } \\
\text { Acompañar en el final de vida }\end{array}$ \\
\hline De prevención & $\begin{array}{l}\text { Vigilar y prevenir caídas cuando se ducha } \\
\text { Vigilar y prevenir caídas cuando va al baño }\end{array}$ \\
& Proteger de relaciones abusivas \\
\end{tabular}

Fuente: Elaboración propia

3 Estas suelen ser las tareas más reconocidas dentro del trabajo de cuidados.

4 Acompañar el final de vida es una tarea referida por mis informantes como muy desgastante, ya que ven sus energías «consumidas» en este proceso y, además, la muerte de la persona a la que cuidan las enfrenta a una situación de incertidumbre laboral.

5 Estas tareas de vigilancia constante provocan problemas de insomnio crónico en las trabajadoras, ya que ven diariamente interrumpido su sueño nocturno por acompañar a los ancianos al baño para vigilar que no se caigan.

6 Las trabajadoras muchas veces intervienen en la relación entre la persona a la que cuidan y sus familiares, protegiéndolas de abusos y maltratos.

7 Para médicos/as y enfermeros/as, las cuidadoras son las fuentes de información más confiable sobre la salud de los ancianos, y son a quienes principalmente van dirigidas las indicaciones terapéuticas. 
Por otra parte, la convivencia jerarquizada en la que se encuentran, además de tener un impacto sobre su dignidad y derechos fundamentales, tiene importantes repercusiones sobre la salud, como aquellas que resultan de la manipulación y control que ejercen los/as empleadores/ as sobre el tiempo libre (escaso o nulo), el descanso, la alimentación, el sostenimiento de los vínculos sociales y la propia autonomía de las trabajadoras, además de la posición de vulnerabilidad en que se las coloca frente a situaciones de maltrato. Respecto a la alimentación, observamos asiduamente situaciones como que los/as empleadores/as las obligan a comer en lugares separados o en sus propias habitaciones, les tiran la comida que las trabajadoras han guardado para ellas, las controlan y juzgan por cuanta comida consumen, les sirven menos cantidad de comida en el plato, lo que las deja con hambre, o las amenazan con dejarlas sin comer. La comida se vuelve entonces en uno de los elementos que conforman los repertorios de demarcación que define Gorbán (2012) como la forma en la que los empleadores trazan límites que intentan demarcar la diferencia social existente entre estos y las trabajadoras.

Después otra vez la hija vino y puso tallarines a la mesa, [...] al marido le dice 'sirve' diciendo, porque todos estábamos con nuestro plato ahí y el marido me sirve, me había puesto seguro un poco más...más me habrá puesto el tallarín, y la hija viene: «así comes todos los días?» me dice.

...yo tomo avena, por el colesterol que tengo...y me hago mi propia avena y un poquitito guardo en la nevera para el día siguiente...tons (sic) vio y lo tiró a la basura, sin decirme nada...

Sandra (57). Perú.

También son frecuentes las situaciones de maltrato, tanto físico como psicológico. En mi experiencia como profesional, he encontrado que las principales perjudicadas, por la agresividad que caracteriza a algunos/as ancianos/as con demencia, son las cuidadoras. Ellas son las más interesadas en que el médico «regule bien» la medicación para evitar la agitación psicomotriz, ya que de otra manera acaban siendo violentadas. Lucía cuenta que mientras la mujer con alzhéimer a la que cuidaba le pegaba, mordisqueaba y arañaba, su empleadora (la hija de la señora) le pedía que le tuviera paciencia. Un día Lucía ya sintió vergüenza de seguir denun- 
ciándole el maltrato de su madre. Ella asegura que soportó esta situación porque sabía que la anciana no quería a otras cuidadoras, lo cual demuestra el grado de responsabilidad que llegan a asumir las trabajadoras respecto a las personas que cuidan. Una situación no poco habitual, ya que las tareas y responsabilidades están por fuera de cualquier contrato en este régimen de internamiento.

Por otra parte, Catalina, además de sufrir la mezquindad e infravaloración por parte de la señora a la que cuida y con quien convive, debe soportar las agresiones y humillaciones por parte del hijo de la señora, quien la culpa de todo y hace comentarios negativos sobre la comida que cocina. También le aflige ser testigo de la violencia que ejerce el hijo sobre su madre (la señora).

Yo ya me cansé, yo muchas veces pensaba: cómo puedo yo después de haber sufrido horrores, pasar tantas situaciones horribles, soportar. Claro, que no es mío, no es mi historia, no es mi...mi problema, no es mi ¿no? pero yo lo estoy viviendo...y lo puedo soportar sin tanto dolor, sin que me afecte tanto...pero ya no.

Catalina (65). Argentina.

Hay que destacar que, si este vínculo jerarquizado se da en un contexto de presunto cariño y afecto mutuo, la relación puede ser percibida por las mujeres como positiva, a veces de una forma tan estrecha como un vínculo de madre e hija, como refería Elena. Me refiero a 'presunto' cariño porque en este tipo de vínculos jerarquizados, las relaciones de poder entre la persona cuidada y la persona cuidadora, si bien variables, pueden aparentar un vínculo maternal o de supuesta amistad cuando de hecho lo que existe es una relación de dominación.

\subsection{Cuidados transnacionales y salud: un vínculo desatendido}

Aunque en ciertos términos la emigración femenina supone una ruptura con el rol tradicional asignado a las mujeres - el cual supone mantenerse en el ámbito de la vida privada, asegurando la crianza de los propios hijos/as y la cohesión de la familia nuclear y extensa- esta no es más que una seudoruptura, ya que en el país de destino les espera un nicho labo- 
ral que las continúa manteniendo en el ámbito doméstico, prácticamente encerradas, realizando las mismas tareas de cuidado. Además, a pesar de la distancia, muchas mujeres continúan siendo las principales responsables de la reproducción de los 'suyos' en el país de origen y las encargadas de asegurar su supervivencia.

No es el objetivo de este trabajo ahondar en las concepciones y subjetividades sobre lo que es o no es la maternidad, ni sobre los determinantes de dicha construcción social. De una manera más pragmática, usaré el concepto de maternidad transnacional (Herrera, 2010) para enmarcar estas prácticas y energías puestas en el cuidado de los hijos e hijas a distancia, de manera que puedan comprenderse sus posibles efectos en la salud. No resulta extraño que, en sociedades donde el vínculo maternofilial es blanco de innumerables mandatos, expectativas y prejuicios, encontremos que la separación entre las mujeres y sus hijos/as de corta edad, por tiempos prolongados, tenga un impacto en la salud de ambas partes. Problemas asociados al estado de ánimo, angustia, ansiedad, insomnio, somatizaciones, dolores crónicos, son solo algunos de los síntomas que he observado con frecuencia en la práctica clínica y he podido corroborar en el trabajo de campo.

En el caso de Elizabeth, Sandra, Catalina, Elena y Lucía, la responsabilidad que tenían hacia su propia vida y la de sus hijos/as fue la que definió la decisión de emigrar, aun teniendo que dejarlos/as al cuidado de otras personas, en algunos casos a temprana edad. Esto fue así en el caso de Lucía, que emigró cuando su hija tenía dos años, o Elizabeth, cuya hija tenía siete años al momento de su partida. El desarraigo por la interrupción de los vínculos afectivos en origen, incluida la separación de los/as hijos/as, tiene fuertes implicaciones sobre la salud, tanto de los/as niños/as como de las mujeres que emigran (Mazarrasa, Martínez, Llacer, \& Castillo Rodríguez, 2004). Esto ocurre, en parte, por la ruptura con el rol tradicional asignado para ellas.

Claro, porque es que una madre... es como si te hubieran arrancado... y este... que no es normal eso para nosotras, que la madre tiene que estar con los hijos, y en esa edad que le he dejado... 
Había un día que era...estaba sentada así, haría dos meses así recién trabajando y empezó a oprimirme así el pecho. Claro, yo estaba sufriendo mucho y dije pues de que yo no he venido a eso, entonces empecé a recordar mi situación de Perú, y digo 'Dios mío, si algo me pasa pues peor, mis hijos ni mi cadáver me verán, tengo que ser fuerte’ empecé a decir que no, que esto no podía consumirme a mí, eso, el sufrimiento que era mucho.

Sandra (57). Perú.

Bastante duro, sí, sí. En primer lugar, porque uno deja a sus seres queridos allí, mi madre, bueno y...mis hijos también porque en el tiempo que yo me vine solo tenía a mi hija mayor por acá y ya he dejado mi hija que dependía de mí, la que tiene discapacidad que es la más pequeña, y todo eso...hombre, me preocupaba muchísimo. Casi ni dormía, me dio un derrame [facial] como a los tres meses de haber llegado por acá.

Elena (47). Honduras.

Es como si algo te faltara... como que no puedes respirar... y cuando te pones, o por lo menos en mi caso cuando estoy de noche es la misma cosa, es como dormir y es como que no durmiera a la vez.

Elizabeth (32). Paraguay.

El ejercicio de la maternidad a distancia resultaba una tarea compleja para mis informantes, y en el caso de Lucía aún más, ya que su hija menor tenía apenas dos años cuando ella partió hacia Barcelona, aunque consiguió volver a verla tres años después, cuando la niña ya tenía cinco años. La dejó a cargo de su hermana, con quien no tenía una buena relación y sufría por parte de ella y su marido muchas extorsiones y estafas. Ella asegura que las malas intenciones de su hermana y los conflictos derivados de esta situación son el motivo de la falta de afecto por parte de su hija y del hecho de que esta no la reconozca plenamente como su madre. Ha tenido que luchar para que no le quiten su apellido a la niña y para reivindicar su lugar de madre a la hora de tomar decisiones importantes, como la de llevar a cabo (o no) rituales religiosos como su comunión. Sin embargo, su hija continúa escribiéndole solo a fin de mes para pedirle las remesas. Desde el principio de su experiencia migratoria, la hija de Lucía 
se convirtió en el elemento de extorsión por parte de su hermana para exigirle dinero. Esta situación, sumada a las estafas de su cuñado, contribuyó tanto a su empobrecimiento en Barcelona, como a su afectación por la mala relación con su hija.

Ella [su hermana] se hizo cargo, que yo le iba a mandar. Le mandaba todos los meses el sueldo y mes que no mandaba... ¡bueno! Al rato me estaba llamando mi hija. Tenía tres o cuatro años por ahí y ya estaba llamando 'que no he comido, que solamente he comido esto' y lloraba....

«¿Dónde te dejo a tu hija?» me dijo [su hermana], «porque yo no tengo espacio pa' tenerla»'»

Lucía (48). Bolivia.

Con el ejemplo de Lucía podemos ver que la gestión de la maternidad transnacional se encuentra fuertemente condicionada por la fortaleza de las redes sociales y las relaciones de intercambio y reciprocidad entre los miembros de la familia en origen (Parella, 2012). Las amenazas de dejar a su hija pequeña en la calle llevaron a Lucía a tener que desarrollar un plan «anticrisis» ${ }^{8}$ para asegurar la vivienda de su hija. Esto implicaba otorgar un préstamo de determinada cantidad de dinero - en este caso fue de veinte mil euros - al propietario de un piso en el país de origen, sin cobrarle interés, a cambio de que este último les alquilase su piso sin cobrar renta. Lucía realizó esta maniobra con su cuñado como intermediario, pero este finalmente la estafó, por lo cual ella perdió todo su dinero y se frustraron los planes de construir su casa en Bolivia para finalmente regresar.

La misma estrategia «anticrisis» utilizó Sandra para solucionar el problema de la vivienda de sus hijos en Perú, pero en su caso con resultados satisfactorios. «iPor teléfono cuántas cosas he solucionado!» afirma. Ella también desarrolló otro tipo de estrategias para ahorrar y cumplir con el objetivo de comprar una casa para ella y sus hijos, como fue la creación de lo que ella llamaba «la junta». Este era un esquema de financiación cooperativo en el que, entre diez mujeres, también de Perú, ponían quinientos euros por mes cada una y luego sorteaban el orden en que le tocaba a cada una cobrar los cinco mil euros mensuales que se juntaban. El cobro se

8 Este término fue utilizado por otra de mis informantes, Sandra, para referirse a la misma estrategia que utilizó Lucía. 
hacía de 'ida y vuelta', cobrando diez mil euros al final de las dos rondas. Este sistema les permitió en un principio pagar las deudas que contrajeron para poder viajar, y posteriormente ahorrar para poder comprarse una casa en el lugar de origen. Para formar parte de «la junta», estaban, de alguna manera, obligadas a trabajar de internas - si bien este no era un requisito explícito- ya que entre los quinientos euros mensuales que tenían que aportar a «la junta» y lo que cada una enviaba de remesas a sus familias (Sandra enviaba otros quinientos euros), solo les quedaba un pequeño resto para sus gastos (alrededor de doscientos euros), de modo que solo era posible sobrevivir trabajando de interna.

Es notable también la preocupación de mis informantes respecto al cuidado de sus hijas en el país de origen, ya sea por el miedo a que sean abusadas, o a que se embaracen y «se destruyan la vida», como refería Sandra. También las moviliza el deseo de estar acompañando a sus hijas mientras atraviesan cambios de etapas, como el momento de la pubertad, que es cuando piensan que más las van a necesitar. En el caso de Elizabeth, esto influye en la planificación de su fecha de retorno.

La niña cuando empieza a tener las reglas, que siempre está... porque una sobrina mía siempre se ha criado con mi hermano y hasta que su padre no le dijo que qué le pasaba, ella estaba asustada, no sabía qué decir, pensaba que su padre se iba a enfadar en todo, hasta que se ha ido mi madre junto a ella y explicarle las cosas, que eso eran naturales, cosas que sí o sí podían pasar. La otra que se ha embarazado muy pronto, tal vez porque dijo, bueno son excusas que ponen, pero... bueno tal vez son reales, tal vez porque sus madres no le habían dicho que tener una relación no era para tener un hijo, por lo menos al menos siendo tan joven. Entonces pues, esa temporada yo la quiero vivir.

Elizabeth (32). Oriunda de Paraguay.

En el peor de los panoramas, a las mujeres también les toca afrontar la muerte de familiares a distancia, y a Elena le tocó vivirlo con uno de sus hijos. El desconsuelo por la pérdida intensifica los sentimientos de culpa por lo que entienden como 'el abandono' de sus hijos al emigrar. Con estos sentires afloran los deseos de volver y se reconfiguran las prioridades. 
Más que todo que he entendido pues que mis hijos que tenía allá también me necesitaban, porque... por teléfono quizás no es lo mismo, y digo yo, todo el tiempo que desperdicié con mi hijo... estando él allá y yo acá...tantas cosas ¿me entiendes? El año pasado, que él me decía que fuera a pasar la Navidad con él y no he ido por estar con el trabajo, y el trabajo, y el trabajo. Le dije 'no hijo, después voy a ir' y mira... después ya no lo volví a ver con vida. Entonces digo yo antes me voy a ir allá a estarme un tiempo a quedarme allá con el que tengo.

Elena (47). Oriunda de Honduras.

Es notable la escasa consideración respecto al impacto de la maternidad transnacional en la salud de las mujeres migrantes, ya que ha sido sorprendentemente subestimado en la literatura hasta ahora. Esto se debe, quizás, al cierto recelo por asumir que el vínculo maternofilial sea universalmente relevante a la hora de hablar de la salud de las mujeres, lo que provoca que, finalmente, se esencialice el rol de la mujer como cuidadora innata. En este sentido considero que un enfoque interpretativo de las narrativas da lugar a que las mujeres puedan expresar la importancia y el significado que el vínculo maternofilial tiene para ellas, resolviendo el problema de un potencial sesgo. No abordarlos implica, por el contrario, mantener oculta una importante dimensión del sufrimiento de muchas mujeres, como señalan Hondagneu-Sotelo \& Avila:

While demanding the right for women workers to live with their children may provoke critiques of sentimentality, essentialism, and the glorification of motherhood, demanding the right for women workers to choose their own motherhood arrangements would be the beginning of truly just family and work policies, policies that address not only inequalities of gender but also inequalities of race, class, and citizenship status (1997: 568). 


\section{Conclusiones}

La salud de mujeres latinoamericanas que trabajan como cuidadoras en Barcelona atraviesa una situación que se caracteriza por una multiplicidad de violencias y desigualdades, favorecidas estructuralmente por políticas de Estado patriarcales y de raíces coloniales. Las leyes españolas colocan a sujetos migrantes racializados en una situación de ilegalidad que les impide gozar de derechos fundamentales. En el caso de las mujeres migradas, despojadas de su tierra y sus vínculos afectivos, se le suman las relaciones de opresión por su condición de mujer, su situación de empobrecimiento y su origen étnico, en un sistema global de jerarquías raciales donde América Latina continúa representando — no solo simbólicamente - una colonia española y sus ciudadanas son naturalizadas como parte de una servidumbre moderna. Esta posición de subordinación social en donde se coloca a este colectivo de mujeres tiene un impacto en su salud y en su acceso al sistema sanitario, razón por la cual este trabajo se propone exponer sus padecimientos y preocupaciones.

Más allá de las discrepancias respecto a la definición y los límites de esta dimensión tan grande dentro del trabajo reproductivo que son los cuidados, podemos reconocer que, en tanto que requieren de una gran inversión de tiempo y energía, tienen unos determinados efectos sobre la salud. Estos efectos se explican, en parte, por las características específicas de estas tareas, y en parte también por su falta de reconocimiento. A su vez, en el contexto social y político adverso en el que se encuentran, la carga psíquica que implica asumir la responsabilidad, no solo de la propia supervivencia, sino también de los/as hijos/as y otros miembros de la familia o comunidad, tiene repercusiones negativas en su salud mental. $\mathrm{Si}$, además, no cuentan con vínculos afectivos basados en la reciprocidad, tanto en origen como en destino, se enfrentan a un mayor riesgo de empobrecimiento. A esta situación se le agrega que muchas se encuentran trabajando de internas de lunes a lunes, cumpliendo con complejas tareas de cuidado y con escasas posibilidades de desarrollar vínculos significativos o redes de autoayuda en destino (por la casi ausencia de tiempo libre o de descanso) y sobrellevando complejas relaciones con la persona a la que cuidan y sus familias. Debido a estas circunstancias, no nos debe 
resultar extraño el hecho de que algunas de ellas no puedan dormir por las noches, sufran de cansancio crónico o que cada tanto tengan dolores en el pecho, un nudo en la garganta o palpitaciones.

En estas circunstancias debemos preguntarnos cómo desarticulamos la naturalización de la explotación de mujeres latinoamericanas, justificada por las necesidades de cuidados de personas mayores en países europeos, además de lo ya cuestionado por Pérez Orozco (2010): ¿cómo arribamos a un régimen global de cuidados justo? Resulta necesario profundizar en el estudio y la visibilización de sus condiciones de vida, así como en las distintas dimensiones en las que su existencia es precarizada. Tanto la explotación laboral que padecen, como las tareas de cuidado transnacional que realizan son factores que deben ser considerados en futuras investigaciones que se propongan un abordaje de su salud de manera realmente integral. El no reconocimiento de los vínculos transnacionales implicaría, además, negar una dimensión vital de la existencia y la salud: el de la afectividad y sus determinantes socioculturales.

En este sentido, un enfoque interpretativo y crítico de la salud de este colectivo que recupere sus narrativas de aflicción posibilita la expresión de la importancia y el valor que las mujeres atribuyen a sus vínculos en general, y al vínculo maternofilial en particular. De igual modo, resulta imprescindible abordar la salud de las mujeres desde una perspectiva feminista, para comprender de qué manera el padecimiento que afecta a estos cuerpos es también un reflejo de un sistema global desigual que pide a gritos el reconocimiento económico y social de las tareas de cuidado, así como su redistribución — con plena participación masculina y responsabilidad estatal - para avanzar hacia una mayor justicia social y equidad de género. 


\section{Bibliografía}

Ahonen, E. Q.; López, M. J.; VÁzquez, M. L.; Porthé, V.; Gil-González, GarCÍA, A. M.; Benvides, F. G. (2010). «Invisible work, unseen hazards: the health of woman immigrant household service workers in Spain». American Journal of Industrial Medicine, 53 (4), 405-416.

Bettio, F.; Simonazzi, A., \& Villa, P. (2011). «Cambios en los regímenes de cuidados y migración femenina. El 'Care Drain' en el mediterráneo» (pp. 305-335). C. Carrasco, C. Borderías, \& T. Torns (eds.). El trabajo de cuidados. Historia, teoría y políticas. Madrid: Catarata.

Bover, A.; Taltavull, M.; Gastaldo, D.; Luengo, R.; Izquierdo, M. D.; JuanDO-PRATS, C.; Robledo, J. (2015). «Calidad de vida de trabajadoras inmigrantes latinoamericanas como cuidadoras en España». Gaceta sanitaria, 29 (2), 123-126.

Carrasco, C.; Borderías, C. \& Torns, T. (2011). «Introducción. El trabajo de cuidados: antecedentes históricos y debates actuales» (pp. 13-96). C. Carrasco, C. Borderías, \& T. Torns (eds.). El trabajo de cuidados. Historia, teoría y políticas. Madrid: Catarata.

Comas D’Argemir, D. (1995). Trabajo, género, cultura. La construcción de desigualdades entre hombres y mujeres. Barcelona: Icaria.

Comas D'Argemir, D. (2014). La crisis de los cuidados como crisis de la reproducción social. Las políticas públicas y más allá. Periferias, fronteras y diálogos. Actas del XIII Congreso de Antropología de la Federación de Asociaciones de Antropología del Estado Español, Tarragona, 2-5/09/2014, Universitat Rovira i Virgili, 329-349.

Dobrée, P. (2016). Apuntes para una aproximación conceptual al cuidado desde la perspectiva de la antropología. (pp. 172-184). ONU Mujeres \& KRISCHKe, P. ¿Por qué nos preocupamos por los cuidados? UN Woman Training Center.

Esteban, M. L. (2017). «Los cuidados, un concepto central en la teoría feminista: aportaciones, riesgos y diálogos con la antropología». Instituto Català d'Antropologia, 22 (2), 33-48.

FEDERICI, S. (2018). Calibán y la bruja. Mujeres, cuerpo y acumulación originaria. Madrid: Traficantes de sueños. 
Gorban, D. (2013). «El trabajo doméstico se sienta a la mesa: la comida en la configuración de las relaciones entre empleadores y empleadas en la ciudad de Buenos Aires». Revista de Estudios Sociales, (45), 67-79.

Herrera, G. (2010). «Stratified workers/stratified mothers: Migration policies and citizenship among Ecuadorian inmigrant women» (pp. 55-76). W. Chavkin, \& J. Maher (eds.). The globalization of motherhood. Deconstructions and reconstruction of biology and care. Routlegde: Oxon.

Hochschild, A. (2002). "Love and Gold» (pp. 15-30). Ehrenreich, B. \& Hochschild, A. (eds.). Global Woman. Nannies, maids and sex workers in the new economy. Nueva York: Holt Paperback.

Hondagneu-Sotelo, P.; Avila, E. (1997). "I'm here but I'm there: the Meanings of Latina Transnational Motherhood». Gender and Society, 11 (5), 548-571.

Hondagneu-Sotelo, P. (2001). Doméstica. Immigrant workers cleaning \& caring in the shadows of affluence. California: University of California Press.

KLeInman, A. (1988). The illnes narratives. Suffering, healing and the human condition. New York: Basic Books.

Kleinman, A.; Eisenberg, L. , \& GOOD, B. (1978). «Culture, Illness and Care. Clinical lessons from Anthropologic and Cross-Cultural Research». Annals of Internal Medicine, 88 (2), 251-258.

Ley orgánica 4/2000, de 11 de enero, sobre derechos y libertades de los extranjeros en España y su integración social. Jefatura del Estado, Boletín Oficial del Estado número 10, de 12 de enero de 2000. (18 de marzo de 2020). Recuperado de: <http://www.exteriores.gob.es/Portal/es/ServiciosAlCiudadano/InformacionParaExtranjeros/Documents/LEY\%20 ORG\%C3\%81NICA\%2042000\%20DE\%2011\%20DE\%20ENERO.pdf>.

Malhotra, R.; Arambepola, C.; Tarun, S.; De Silva, V., \& Kishore, J. (2013). «Health issues of female foreing domestic workers. A systematic review of the scientific and gray literature.» International Journal of Occupational and Environmental Health, 19 (4), 261-277.

Martínez-Hernáez, A. (2000). What's Behind the Symptom? On Psychiatric Observation and Anthropological Understanding. Amsterdam: Harwood Academic Publishers. 
Martínez Hernáez, A. (2015). Antropología Médica. Teorias sobre la cultura, el poder y la enfermedad. Barcelona: Anthropos.

Mazarrasa, L.; Martínez, M. L.; Llacer, A., \& Castillo Rodríguez, S. (2004). Caracterización de algunos problemas de salud de las mujeres inmigrantes en España y de sus determinantes (pp. 161-192). P. DE VILLOTA (ed.). Globalización y desigualdad de género. Madrid: Sintesis.

MenéndeZ, E. L. (2005). «El modelo médico y la salud de los trabajadores». Salud Colectiva, I (1), 9-32.

Morero Beltrán, A., \& Ballesteros Pena, A. (2014). «Las consecuencias de la aplicación del Real Decreto-Ley de Regulación Sanitaria R 16/2012 sobre la salud de las mujeres inmigradas». Investigaciones feministas, 5, 317-341.

Offenhenden, M. (2017). Si hay que romperse una, se rompe. El trabajo de hogar y la reproducción social estratificada (Tesis doctoral). Universitat Rovira i Virgili, Tarragona.

PARella, S. (2004). «Reclutamiento de trabajadoras inmigrantes en las empresas de servicios de proximidad en el Área Metropolitana de Barcelona». Revista española de investigaciones sociológicas, 1 (108), 179-200.

Parella, S. (2007). «Los vínculos afectivos y de cuidado en las familias transnacionales. Migrantes ecuatorianos y peruanos en España». Migraciones Internacionales, 4 (2), 151-188.

Parella, S. (2012). «Familia transnacional y redefinición de los roles de género. El caso de la migración boliviana en España». Papers, 97 (3), 661-684.

Pérez Orozco, A. (2010). «Cadenas globales de cuidados. ¿Qué derechos para un régimen global de cuidados justo?». Instituto Internacional de Investigaciones y Capacitación de las Naciones Unidas para la Promoción de la Mujer(UN-INSTRAW). 